\title{
$3 D$ terahertz beam profiling from two color laser induced plasma with different focusing
}

\author{
A.A. Ushakov ${ }^{1,2,3,4}$, M. Matoba ${ }^{4}$, N. Nemoto ${ }^{4}$, N. Kanda ${ }^{5,6}$, K. Konishi ${ }^{7}$, \\ V.A. Andreeva ${ }^{2,3}$, N.A. Panov ${ }^{2,3}$, D.E. Shipilo ${ }^{2,3}$, P.A. Chizhov ${ }^{1}$, V.V. Bukin ${ }^{1}$, \\ M. Kuwata-Gonokami ${ }^{4}$, J. Yumoto ${ }^{4,7}$, O.G. Kosareva ${ }^{2,3}$, S.V. Garnov ${ }^{1}$ and A.B. Savel'ev ${ }^{2,3}$ \\ ${ }^{1}$ A.M. Prokhorov General Physics Institute RAS, Moscow, Russia, ushakov.aleksandr@physics.msu.ru \\ ${ }^{2}$ Physics Faculty, M.V. Lomonosov State University, Moscow, Russia \\ ${ }^{3}$ International Laser Center of M.V. Lomonosov Moscow State University, Moscow, Russia \\ ${ }^{4}$ Department of Physics, The University of Tokyo, Tokyo, Japan \\ ${ }^{5}$ RIKEN Center for Advanced Photonics, RIKEN, Tokyo, Japan \\ ${ }^{6}$ Photon Science Center, The University of Tokyo, Tokyo, Japan \\ ${ }^{7}$ Institute for Photon Science and Technology, The University of Tokyo, Tokyo, Japan
}

Profiling of the Terahertz (THz) beam emerging from the two color laser plasma interaction gain a lot of attention last years, providing insight into the physics of such an interaction and being of crucial importance for future applications. Beam profiling was demonstrated using electro-optical sampling [1], or using 2D THz camera for 3D measurements [2]. Later the beam profiling for different $\mathrm{THz}$ spectrum components [3] was demonstrated. However, impact from focusing conditions of the two color laser beam - the key parameter of the $\mathrm{THz}$ source in terms of directional output of $\mathrm{THz}$ radiation - has not been assessed yet.

In our paper we described the $3 \mathrm{D}$ beam profiling by $2 \mathrm{D}$ cross-section measurements of $\mathrm{THz}$ radiation from the twocolor laser induced air plasma by using $\mathrm{THz}$ camera [4]. Laser pulses (central wavelength $800 \mathrm{~nm}$, pulse duration $35 \mathrm{fs}$, pulse energy $2.7 \mathrm{~mJ}$, repetition rate $1 \mathrm{kHz}$ ) after the BBO crystal (I-type, $400 \mu \mathrm{m}$ thickness, $13 \%$ conversion efficiency), group velocity dispersion compensator plate and phase plate (half wave plate for fundamental wave and wave plate for second harmonic) were linearly polarized and collinear. Thereafter they were focused by off axis parabolic mirrors with the focal length of 2,4 or 7.5 inches. For the depicting of plasma source to the $\mathrm{THz}$ camera two Tsurupica-lenses with focal length of $10 \mathrm{~cm}$ were used. 2D THz intensity profile measurements have been done after the two lenses with $1 \mathrm{~mm}$ step. For each series of experiments band pass $\mathrm{THz}$ filters (central frequencies $0.6 \mathrm{THz}, 0.8 \mathrm{THz}, 1 \mathrm{THz}, 1.3 \mathrm{THz}, 1.6 \mathrm{THz}$, $1.9 \mathrm{THz}$ ) were used. The sample distributions of $\mathrm{THz}$ radiation are presented in figure 1.

We also conduct measurements of distribution of $\mathrm{THz}$ field from the two color laser induced plasma by using the interferometric technique [5]. For generation and registration of $\mathrm{THz}$ radiation we used laser pulses (central wavelength $800 \mathrm{~nm}$, repetition rate $10 \mathrm{~Hz}$, pulse duration $40 \mathrm{fs}$, pulse energy $2.5 \mathrm{~mJ}$, Gaussian beam di-

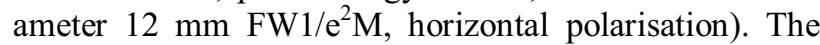
laser radiation was divided into two parts. The main part was used for generation of $\mathrm{THz}$ radiation in the source based on the optical breakdown in air induced by twocolor femtosecond laser pulses [1]. The other part of laser radiation - the probing pulse - after passing through the variable optical delay line was used to measure the electric field strength, as shown in figure 2.
The principle of $\mathrm{THz}$ radiation detection is based on the linear electro-optical effect. The THz pulse and the probing optical pulse $(\lambda=800 \mathrm{~nm})$ were combined on the ZnTe crystal $\left(10 \times 10 \times 0.5 \mathrm{~mm}^{3}\right.$, cut $\left.<110>\right)$. In this case, the polarization of $\mathrm{THz}$ radiation was vertical, while the probing pulse was polarized at the angle $45^{\circ}$. As a result of the electro-optic effect in the optically isotropic $\mathrm{ZnTe}$ crystal the birefringence was induced, and in the above geometry of the experiment the probing radiation in the crystal was split into two waves (with vertical and horizontal polarizations and equal intensities), for which the difference between the refractive indices was linearly dependent on the electric field strength of the $\mathrm{THz}$ pulse.

The nonuniform distribution of the field strength over the crystal cross section caused the nonuniform distribution of the phase difference between the two waves at the output from the crystal. This phase difference was extracted by using an interferometer with polarizer and display of ZnTe crystal surface onto the CCD matrix was realized by telescope.

During the measurements we obtained interferograms in the presence of a $\mathrm{THz}$ pulse (signal) and in its absence (reference). To increase the signal-to-noise ratio for each delay time between the optical pulse and the $\mathrm{THz}$ one, 50 signal and background records (frames) were acquired. The phase was reconstructed by processing the interferograms by means of the method based on filtration in the Fourier-space [6]. As a result of the processing we obtained a two-dimensional map of the mean phase shift change caused by the presence of the $\mathrm{THz}$ pulse field. From the phase shift the $\mathrm{THz}$ electric field strength $\mathrm{E}_{\mathrm{THz}}$ can be extracted [5].

In these experiments the depicting of plasma was realized by using $6 \mathrm{~cm}$ and $15 \mathrm{~cm}$ PTFE Teflon lenses. The spatio-temporal profiles of the $\mathrm{THz}$ pulse electric field, obtained in the experiments, are presented in figure 3 . The electro-optical crystal was placed in the plane, separated by the distance of $1 \mathrm{~cm}$ from the geometric focal point of the PTFE-lens $(\mathrm{f}=15 \mathrm{~cm})$. Because in this region the focused $\mathrm{THz}$ radiation has a spherical wave front, the profiles of the field strength $\mathrm{E}_{\mathrm{THz}}$ have the shape of rings that correspond to the sections of the spherical wave front of the $\mathrm{THz}$ radiation by the planar wave fronts of the probing pulse. With growing delay time (i.e., for later 
arrival of the probing optical pulse) the ring diameter decreases, collapsing into a spot when the planar front of the probing pulse is tangent to the converging spherical front of the THz pulse.
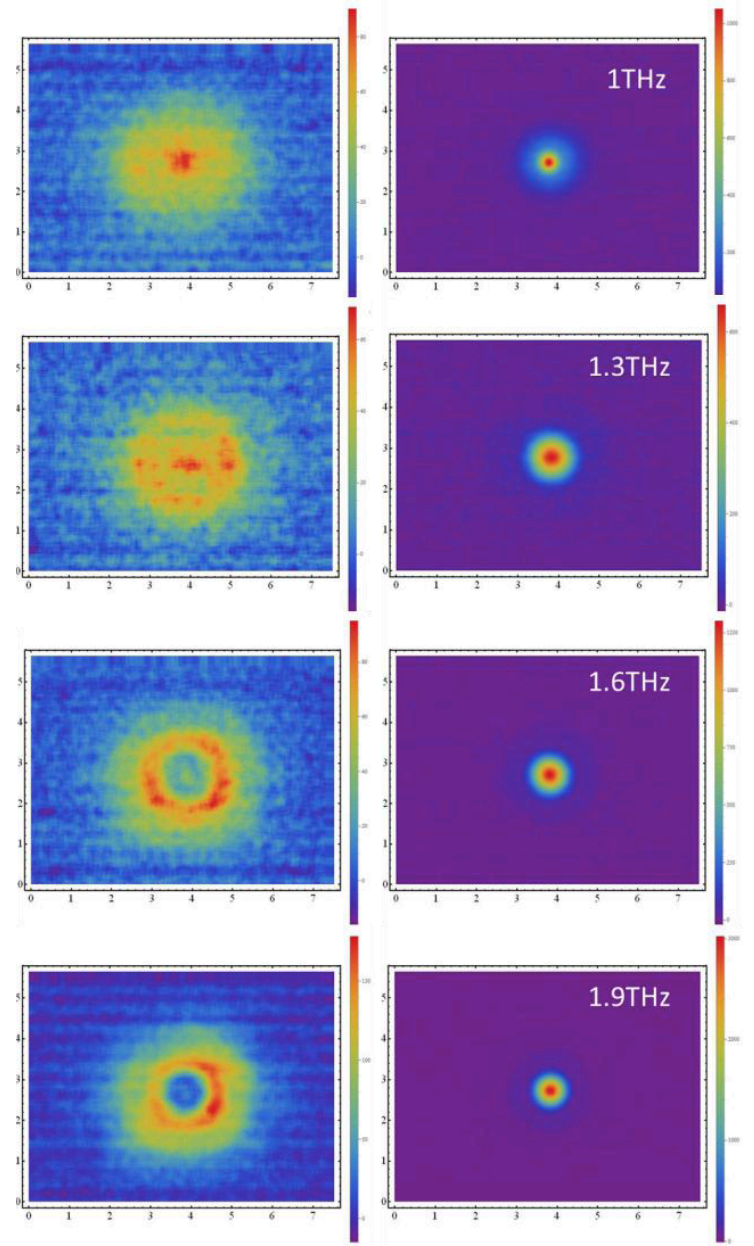

Fig. 1. THz Intensity distribution (at different frequencies) for focused by 4 inches parabola (right) and unfocused (left) THZ beams

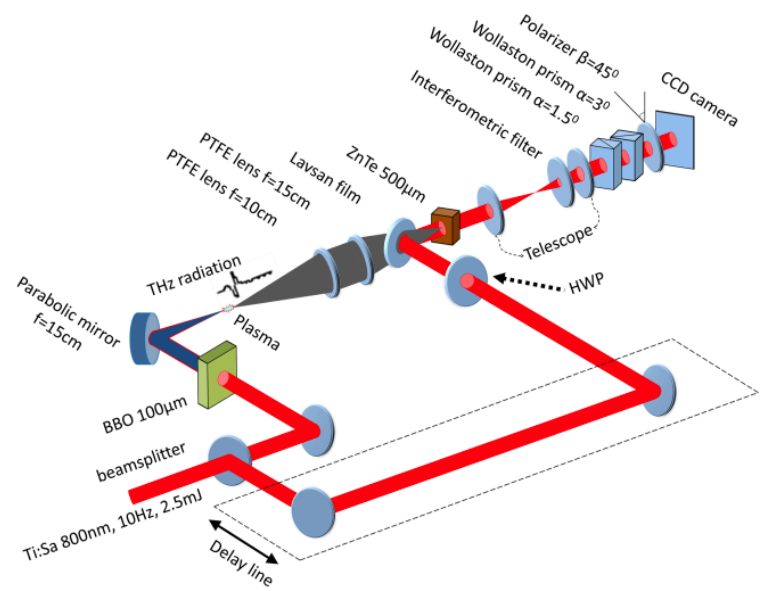

Fig. 2. Scheme of the experimental setup for $\mathrm{THz}$ field distribution measurements

Finally we presented theoretical explanation of the data observed using unidirectional pulse propagation equation [7]. To compare with experimental data $\mathrm{THz}$ beam propagation through the optical system was also included in the simulations. Simulations have demonstrated that aperture effect of optical elements plays an important role in $\mathrm{THz}$ beam profiling and the ring structure is the result of diffraction on the lenses.

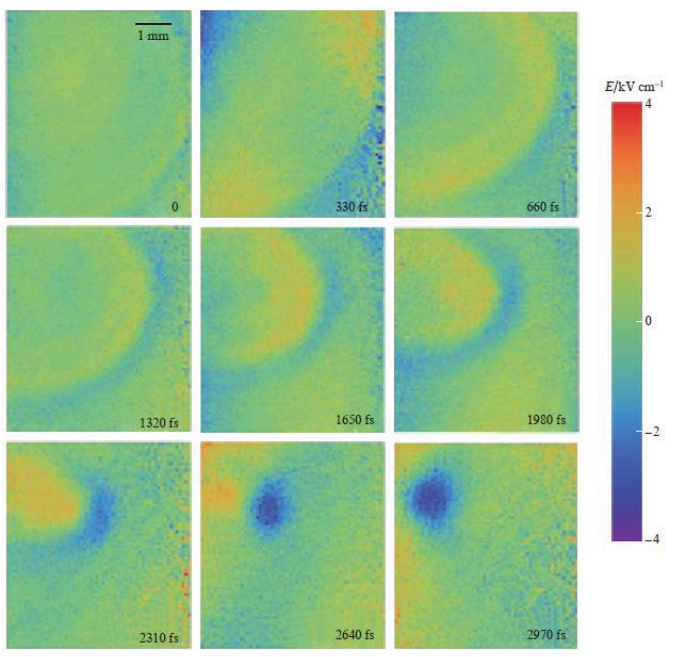

Fig. 3. Images of the spatial distribution of the THz pulse electric field strength at different time delays

This work in part dealing with $\mathrm{THz}$ measurements from femtosecond filaments was supported by the Students and Researchers Exchange Program in Sciences (STEPS) and RSF grant \#16-42-01060, another part of work was supported by RF President's Grant Council (Support to the Leading Scientific Schools programs, Grant No. NSh-9695.2016.2), Grant of RF President for state support of young Russian scientists (Grant No. MK8562.2016.2).

\section{References}

1. Zhong H., Karpowich N., Zhang X.C. Terahertz emission profile from laser induced air plasma. // Appl. Phys. Lett. 2006. V. 88, P. 261103-1-4.

2. Klarskov P., Strikwerda A.C., Iwaszczuk K., Jepsen P.U. Experimental three-dimensional beam profiling and modeling of a terahertz beam generated from a two-color air plasma // New Journal of Phys. 2013. V. 15. P. 075012.

3. Klarskov P., Zalkovskij M., Strikwerda A.C., Jepsen P.U. Spectrally resolved measurements of the terahertz beam profile generated from a two-color air plasma // Proc. of CLEO:2014. 2014. 6-7.

4. Nemoto N., Kanda N., Imai R., Konishi K., Miyoshi M., Kurashina S., Sasaki T., Oda N., Kuwata-Gonokami M. HighSensitivity and Broadband, Real-Time Terahertz Camera Incorporating a Micro-Bolometer Array With Resonant Cavity Structure // IEEE Trans. Microw. Theory Tech. 2016. V. 6. No. 2. P. 175182.

5. Chizhov P.A., Ushakov A.A., Bukin V.V., Garnov S.V. Measurement of spatio-temporal field distribution of THz pulses in electro-optic crystal by interferometry method // Quantum Electron. 2015. V.45. No. 5. P. 434-436.

6. Takeda M., Ina H., Kobayashi S. Fourier-transform method of fringe-pattern analysis for computer-based topography and interferometry // J. Opt. Soc. Am. 1982. V. 72. No. 1. P. 156.

7. Andreeva V.A., Kosareva O.G., Panov N.A., Shipilo D.E., Solyankin P.M., Esaulkov M.N., Gonzalez De Alaiza Martinez P., Shkurinov A.P., Makarov V.A., Berge L., Chin S.L., Ultrabroad Terahertz Spectrum Generation from an Air-Based Filament Plasma // Phys. Rev. Lett. 2016. V. 116, P. 63902. 